Courrier du Centre International Blaise-

Pascal

$28 \mid 2006$

Varia

\title{
Les dernières tribulations des pascalines de Clermont-Ferrand
}

Pierre Pénicaud

\section{(2) OpenEdition}

1 Journals

Édition électronique

URL : https://journals.openedition.org/ccibp/753

DOI : 10.4000/ccibp.753

ISSN : 2493-7460

Éditeur

Centre international Blaise Pascal

Édition imprimée

Date de publication : 16 octobre 2006

Pagination : 37-43

ISBN : 2-84516-305-3

ISSN : 0249-6674

Référence électronique

Pierre Pénicaud, «Les dernières tribulations des pascalines de Clermont-Ferrand », Courrier du Centre International Blaise-Pascal [En ligne], 28 | 2006, mis en ligne le 02 décembre 2015, consulté le 28 juin 2022. URL : http://journals.openedition.org/ccibp/753 ; DOI : https://doi.org/10.4000/ccibp.753

Ce document a été généré automatiquement le 29 septembre 2020.

Centre international Blaise Pascal 


\title{
Les dernières tribulations des pascalines de Clermont-Ferrand
}

\author{
Pierre Pénicaud
}

\section{Historique}

1 Les deux machines arithmétiques surnommées « Pascalines » et appartenant à la Ville de Clermont-Ferrand proviennent directement de la famille de Blaise Pascal. Elles avaient été léguées par Marguerite Périer, fille de Gilberte Pascal et de Florin Périer et donc nièce de Blaise, à l'Oratoire de Clermont. Après la Révolution, la machine dite « de Marguerite Périer ", inv. $\mathrm{n}^{\circ}$ 138, entre dans les collections publiques. Conservée dans différentes institutions, elle est enfin présentée dans les murs de l'hôtel de Fonfreyde, alors musée du Ranquet. Elle y est rejointe par la machine dite « du chevalier DurantPascal » inv. 985-23-1 à 3 (la machine, le coffret et la notice), acquise par la Ville en 1985. Cette dernière, au moment de la Révolution, avait été récupérée par le chevalier Durant-Pascal. 
Portrait de Blaise Pascal (huile sur toile) réalisé post mortem par François II Quesnel (1637-1699) peintre ami des Pascal, à partir de ses souvenirs et du masque mortuaire.

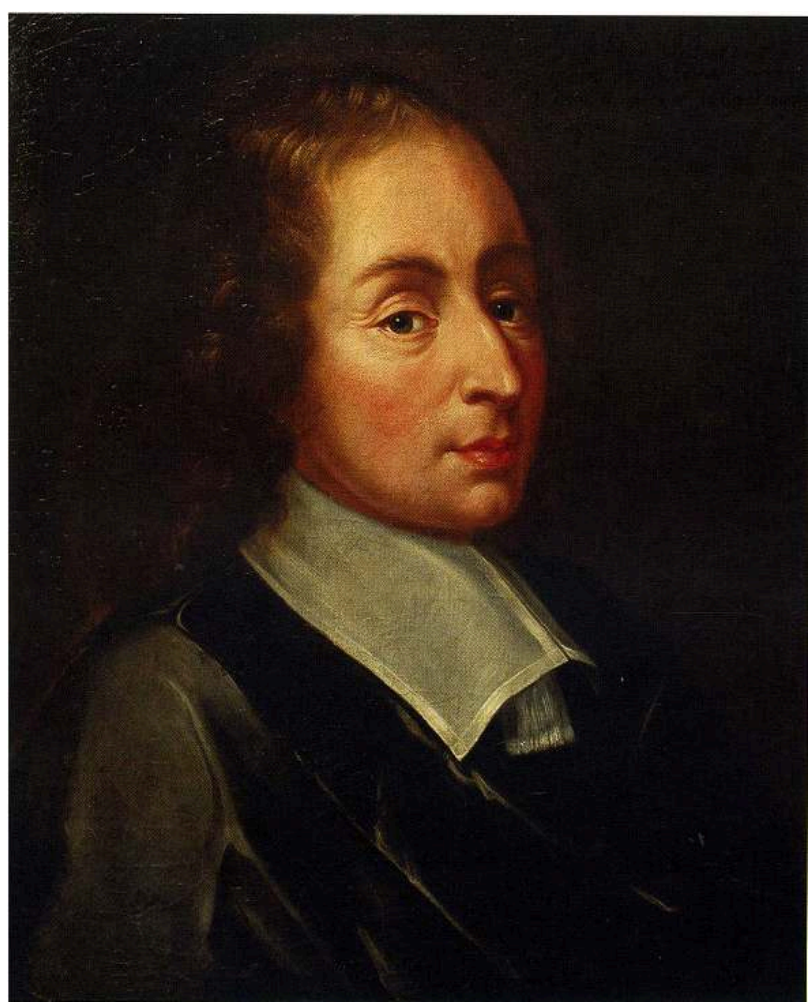

Coll. Musée d'art Roger Quilliot. Cl. A. Jourde. Musée d'art Roger Quillot

Portrait du XVII siècle, anonyme de Gilberte Pascal et épouse de Florin Périer.

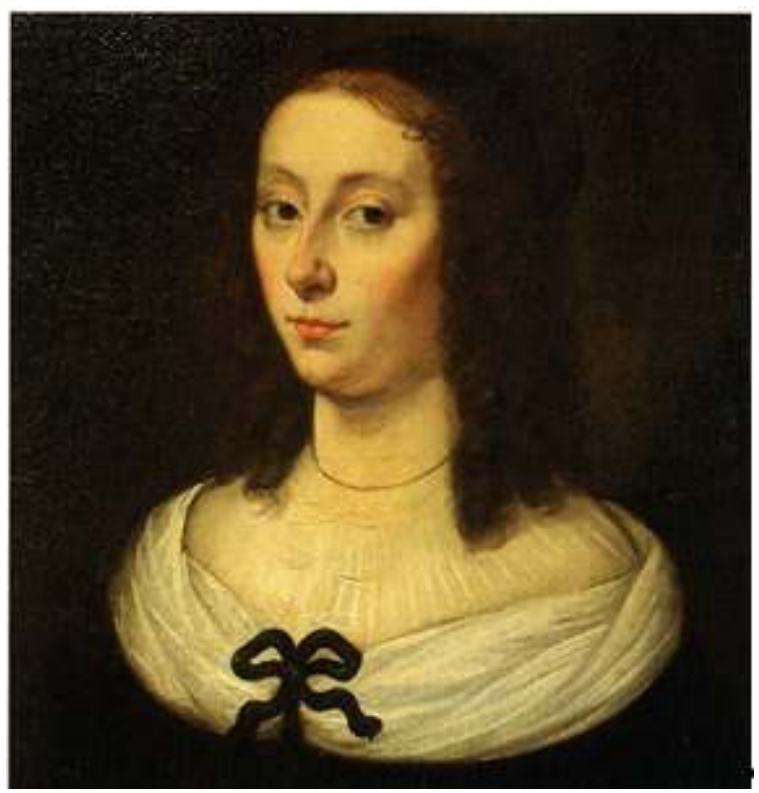

Coll. Musée d'art Roger-Quilliot. cl. A. Jourde.

Musée d'art Roger Quillot 
Armes de la famille Pascal, gravées sur le devant des Pascalines.

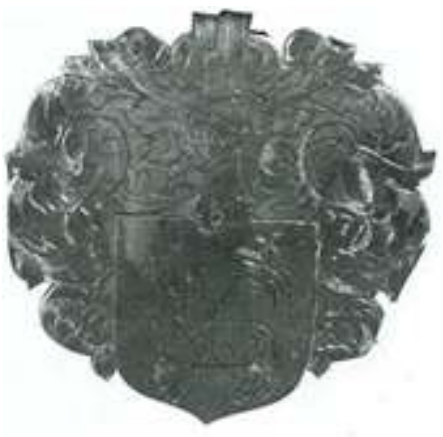

C1. P. de Parédès.

2 En 2001, dans le cadre du projet de rénovation du musée du Ranquet autour d'une thématique culturelle et scientifique basée sur Blaise Pascal et les sciences et techniques, les deux Pascalines sont envoyées au laboratoire du C2RMF (Centre de recherche et de restauration des musées de France) situé dans le palais du Louvre, pour y être étudiées. Malheureusement, des changements de personnels au C2RMF puis l'abandon par la Ville du projet d'un musée Blaise-Pascal, suivi par la décision de fermer définitivement le musée du Ranquet, ont considérablement retardé la mise en route des restaurations et surtout empêché une étude comparative aboutie de plusieurs Pascalines connues, les quatre conservées au musée des Arts et Métiers et celle du musée de Dresde.

3 En 2005, par décision du conseil municipal portant sur le devenir des collections du musée Ranquet, l'ensemble des collections scientifiques et techniques et, avec elles, les deux Pascalines, est affecté au muséum d'histoire naturelle Henri-Lecoq. En effet les collections d'un musée de France, au regard de la loi 2002 dite "loi musée », sont réputées inaliénables et imprescriptibles. Dans le cas d'un musée cessant d'exister, ses collections doivent être affectées dans un autre musée de France. Le muséum d'histoire naturelle Henri-Lecoq voyait donc se créer un nouveau département dans ses collections qui en comportent maintenant cinq: Zoologie, Botanique, Géologie, Sciences et Techniques et Patrimoine écrit.

4 En juin 2005, les Pascalines quittaient enfin le palais du Louvre, restaurées, pour rejoindre Clermont-Ferrand. Elles sont présentées maintenant dans l'ancien hôtel d'Henri-Lecoq parmi les collections du muséum d'histoire naturelle. Ainsi le souvenir de Blaise Pascal se mêle à ceux de deux autres très grands scientifiques, Antoine de Lavoisier et Louis Pasteur.

\section{Les restaurations}

5 La restauration d'un objet de musée est toujours une affaire délicate. En effet, plusieurs impératifs parfois contradictoires rentrent en jeu :

- des impératifs de conservation pour limiter la dégradation naturelle et les interactions entre des matériaux parfois incompatibles ;

- redonner un aspect plaisant à un objet dégradé ;

- conserver à l'objet son authenticité ;

- remplacer les manques, parfois les interpréter. 
6 Quelles que soient les décisions prises, une règle est à respecter : la réversibilité. Dans le cas d'objets complexes comme les Pascalines, plusieurs spécialistes peuvent intervenir, principalement en fonction du matériau.

La pascaline « du chevalier Durant-Pascal », son coffret et la notice avant restauration.

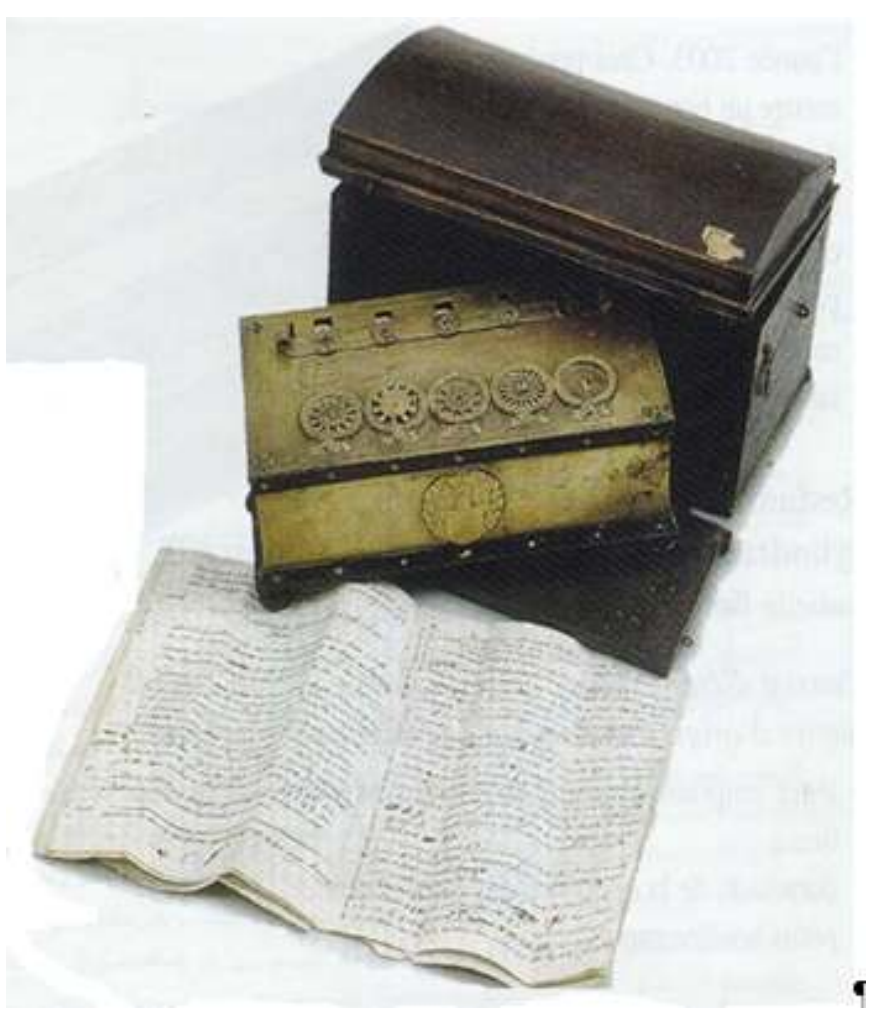

C1. D. Massacrier. 
Détail du coffret de la pascaline « du chevalier Durant-Pascal » après restauration.

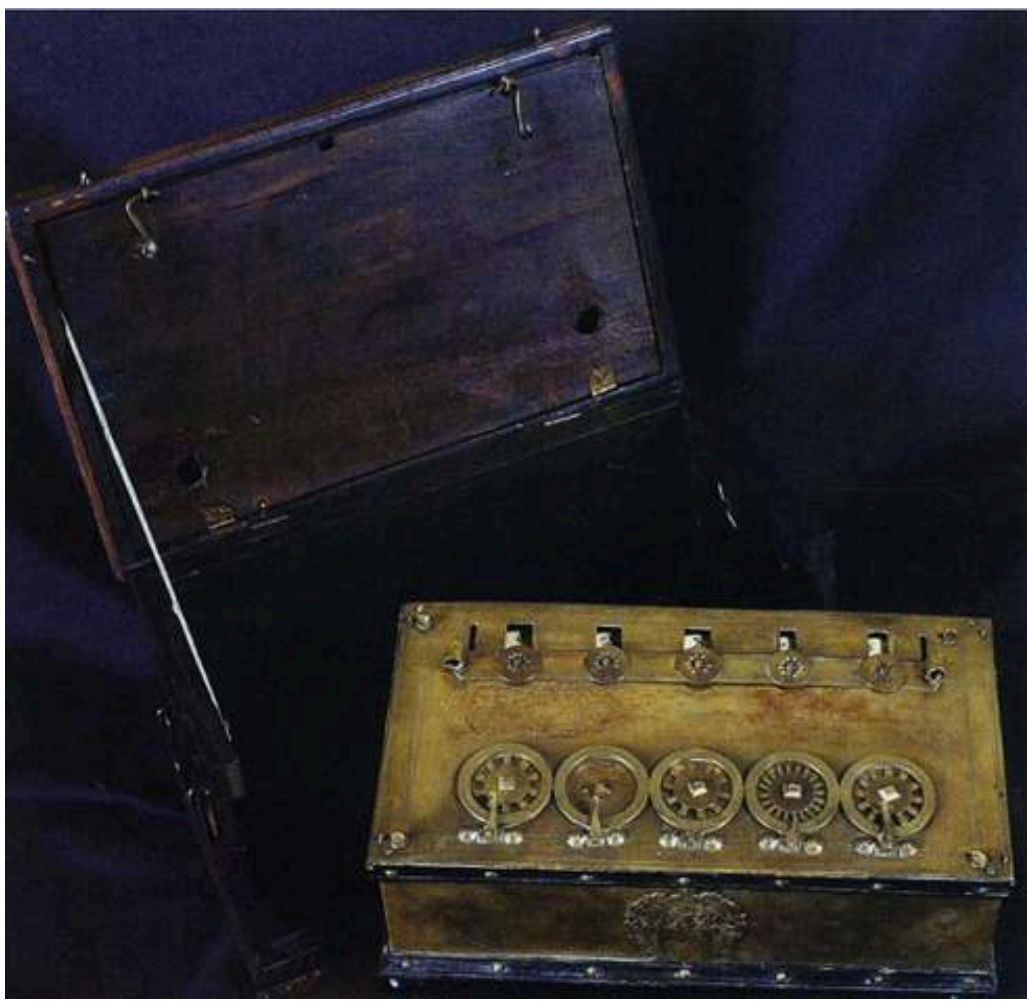

Remarquer la nouvelle position des roues.

C1. P. de Parédès.

Détail du coffret de la Pascaline « du chevalier Durant-Pascal », avant restauration.

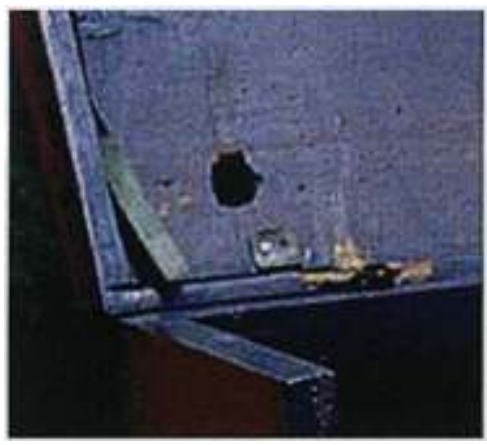

Remarquer les fissures, la moulure, la charnière et le ruban.

Cl. D. Massacrier 
Détail du coffret de la Pascaline « du chevalier Durant-Pascal », après restauration.

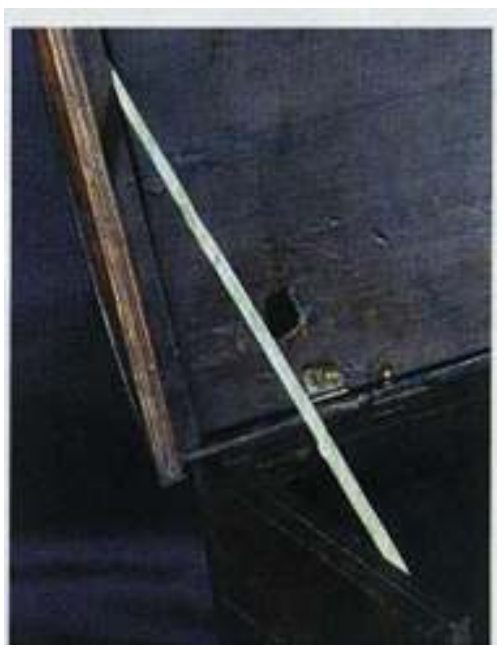

Cl. P. de Parédès

\section{Restauration du métal (caissons en laiton et mécanismes des deux machines).}

7 Olivier Morel, restaurateur.

\section{Constat d'état :}

- des traces de corrosions dues aux contacts des doigts, aux produits de nettoyage, aux graisses anciennes ;

- des parties manquantes, comme des goupilles, des vis, des écrous ;

- des parties abîmées, comme les dents de certains engrenages des indexes et des roues.

\section{Restauration :}

- les deux machines ont été plus ou moins démontées, pour être nettoyées légèrement (en évitant d'enlever le vernis) à la vapeur et par micro-brossage ;

- les écrous, vis et goupilles manquantes ont été refaites d'après moulages des parties existantes et marquées de l'année 2003. Ceci pour permettre un bon maintien des deux mécanismes ;

- les engrenages tordus ont été redressés ;

- pour la protection future, une cire micro-cristalline a été appliquée. 
La Pascaline « de Marguerite Périer » Avant restauration.

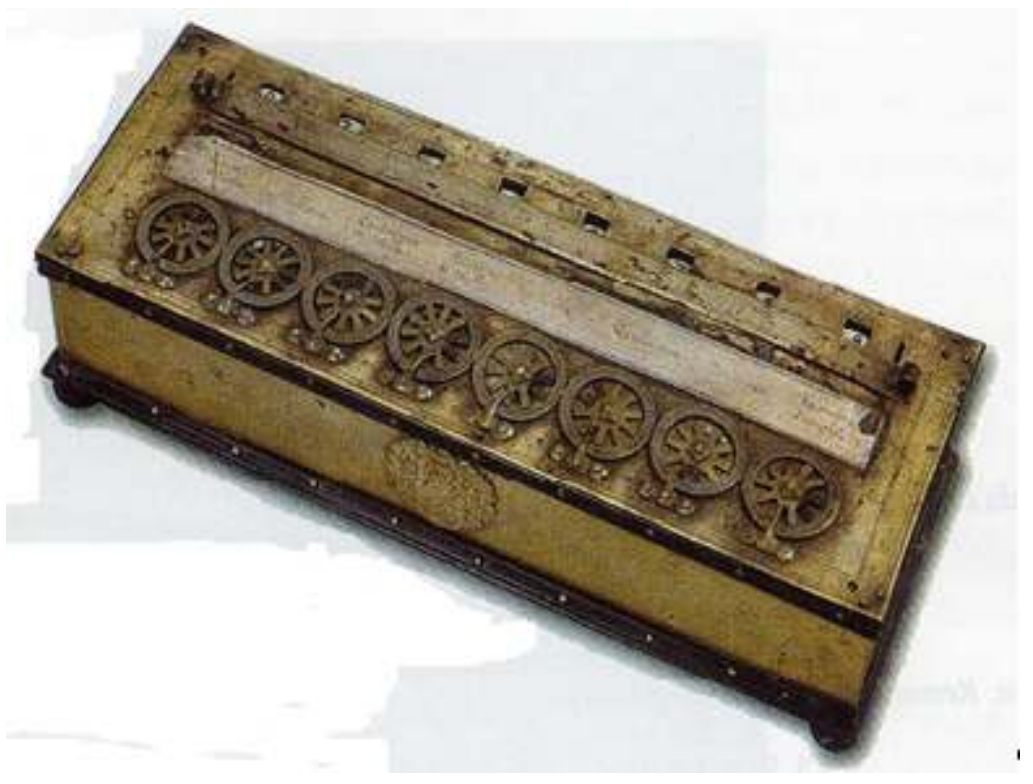

C1. 1. D. Massacrier

Détail de la Pascaline « de Marguerite Périer » avant restauration

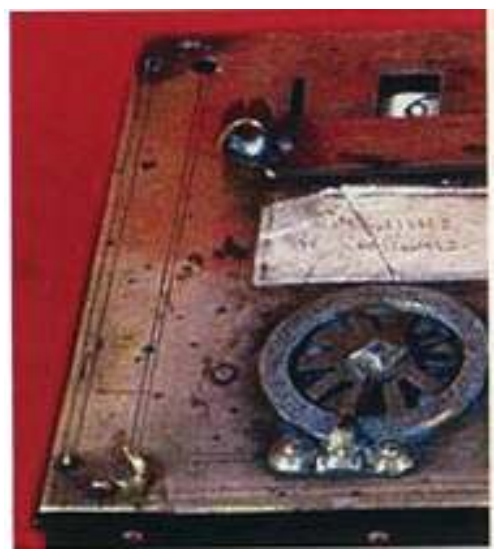

C1. 1. D. Massacrier 
Détail de la Pascaline « de Marguerite Périer » après restauration

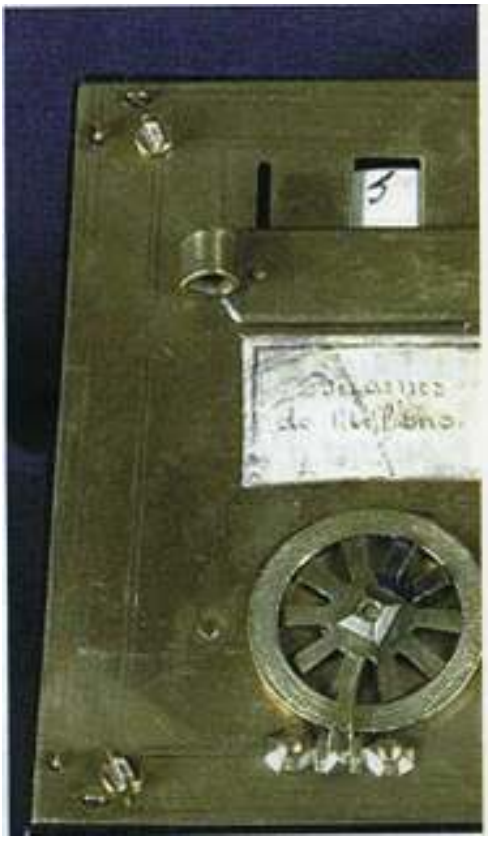

Remarquer les taches sur le laiton, l'oxydation due aux produits d'entretien, la restauration du papier et la nouvelle vis.

Cl. P. de Parédès

La Pascaline « de Marguerite Périer » après restauration

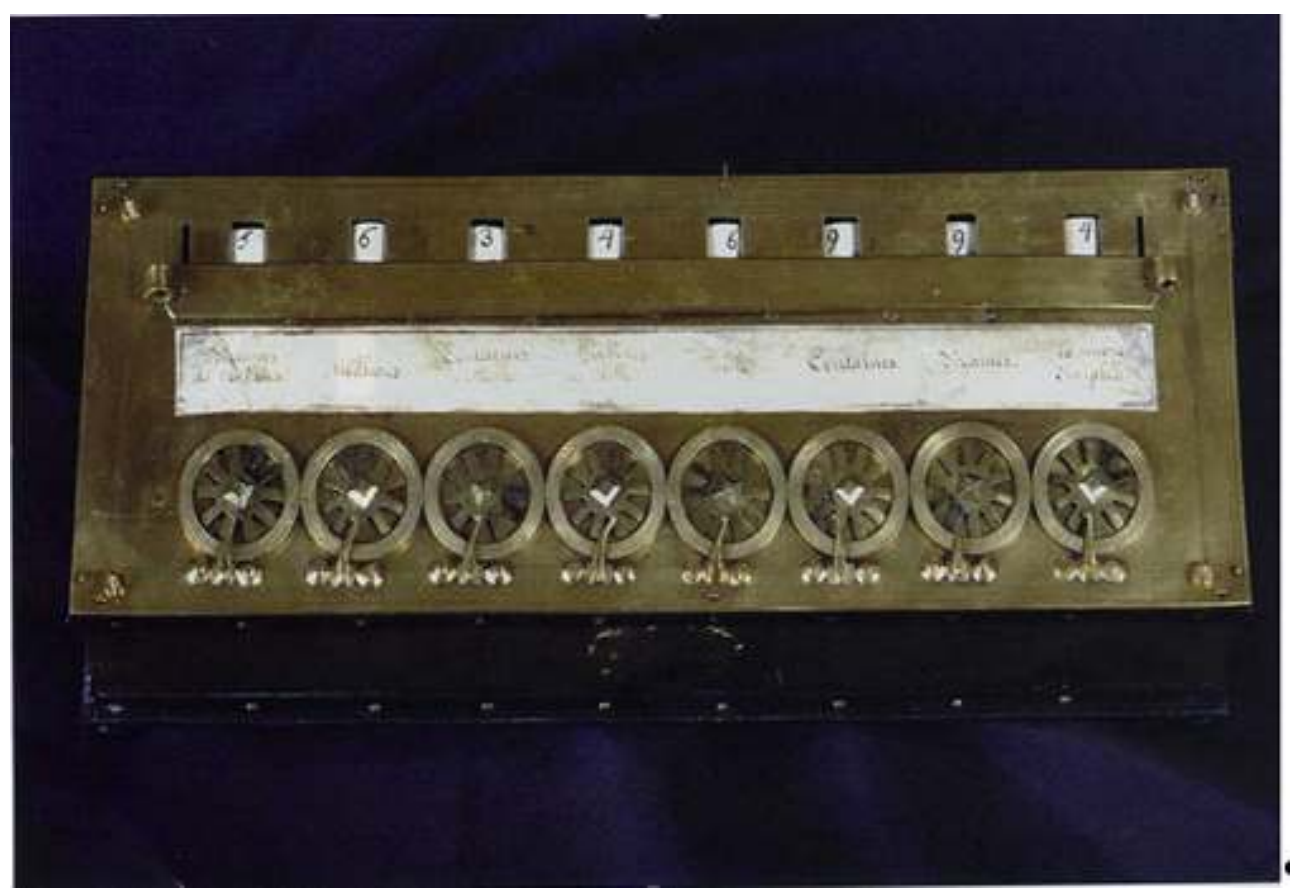

Cl. P. de Parédès 


\section{Restauration du papier (papier des cylindres, bande de papier collé, manuscrit)} conservation général

- léger empoussièrement de surface et piqûres d'oxydation ;

- corrosion de la cellulose des parties encrées ;

- petits soulèvements.

\section{Restauration :}

- dépoussiérage à la gomme ;

- consolidation à la colle de méthylcellulose.

13 Constat d'état : bande de papier collée sur la machine «de Marguerite », daté entre 1850-1950, vélin de mauvaise qualité et abîmé.

- Surface salie et tachée;

- collage non uniforme ;

- cassures et une lacune.

Première page de la notice accompagnant la Pascaline « du chevalier Durant-Pascal ».

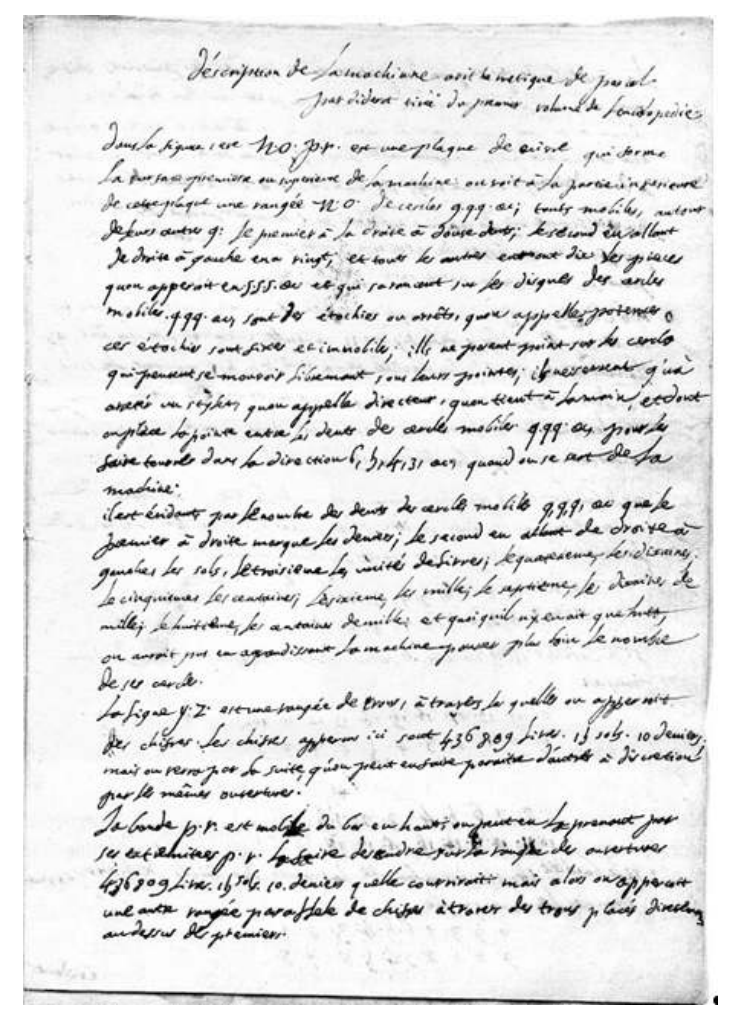

\section{APRÈS RESTAURATION}

C1. musée Lecoq.

\section{Restauration :}

- Décollage de la bande et nettoyage ;

- consolidation des cassures et comblement de la lacune avec papier-japon ;

- doublage de la bande de papier alors collée sur le plateau. 
Constat d'état : manuscrit, papier du XVIII ${ }^{e}$ siècle, plié en trois.

- Corrosion due à l'encre ;

- déchirures et lacunes ;

- large tache ;

- pliures.

\section{Restauration :}

- Mise à plat sans démontage ;

- dépoussiérage léger ;

- consolidation et comblement des déchirures et lacunes avec papier-japon.

\section{Restauration du tissu (ruban de soie permettant de maintenir ouvert le coffret de la machine « du Chevalier Durant-Pascal »}

\section{Restauration :}

- restauration des charnières ;

- collage et comblement des fissures ;

- réalisation de la moulure manquante ;

- retouche colorimétrique et finition.

- désolidarisé du coffret ;

- rompu et incomplet ;

- fragilisé, replié et cousu.

Antoine Buisson, restaurateur.

- la fixation des charnières détériorée ;

- fentes dans le bois et assemblages fragilisés ;

- moulure manquante.

- suppression des coutures et mise à plat (par humidité) ;

- confection d'un ruban de taffetas et fixation de l'ancien sur le nouveau par couture ;

- ce nouveau dispositif a été refixé à l'identique sur le coffret par le restaurateur bois.

\section{Restauration du bois (le coffret de la machine « du Chevalier Durant- Pascal »)}

La restauration des machines a permis quelques études plus poussées, en particulier sur le papier. Ainsi les cylindres sont d'origine, la comparaison graphique des chiffres permet de préciser que les cinq cylindres de la machine " du chevalier Durand-Pascal » ont été réalisés en même temps et écrit par la même personne (4 et 8). Le papier et l'encre des cylindres de la machine « de Marguerite Périer " semblent similaires, d'où l'on peut penser que ces deux machines ont été construites à peu près en même temps. En revanche, la graphie démontre que sur cette dernière machine, c'est une autre personne qui a écrit les chiffres. La bande de papier collée sur la machine «Marguerite Périer » l'a été récemment, alors que la machine était déjà dans les collections 
publiques! Il était déjà connu que le manuscrit était une copie de la notice, mais l'étude confirme que cette copie est ancienne (XVIII siècle). Elle a permis aussi de replacer des éléments qui au cours des temps avaient été mélangés, comme les roues dentées du plateau enlevant la cohérence du fonctionnement des machines.

Comparaison des graphismes des chiffres sur les rouleaux de la machine " du chevalier DurantPascal ». Cylindres 5 et 4 , et 4 et 3
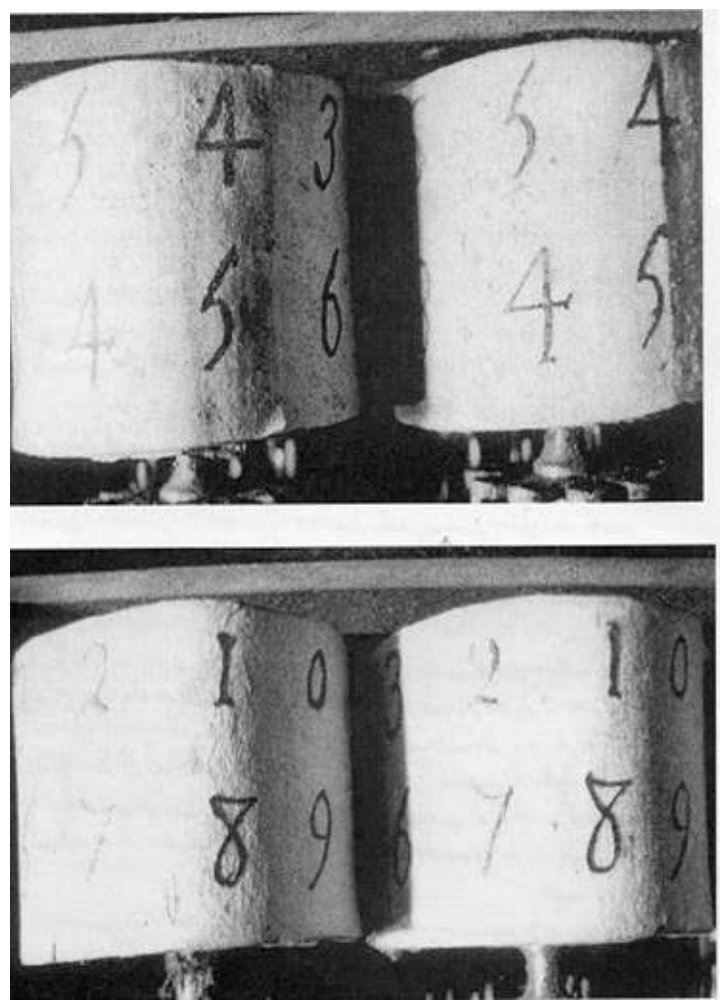

Cl. I. Bonnard.

Cylindre 2 de la machine « du chevalier Durant-Pascal ».

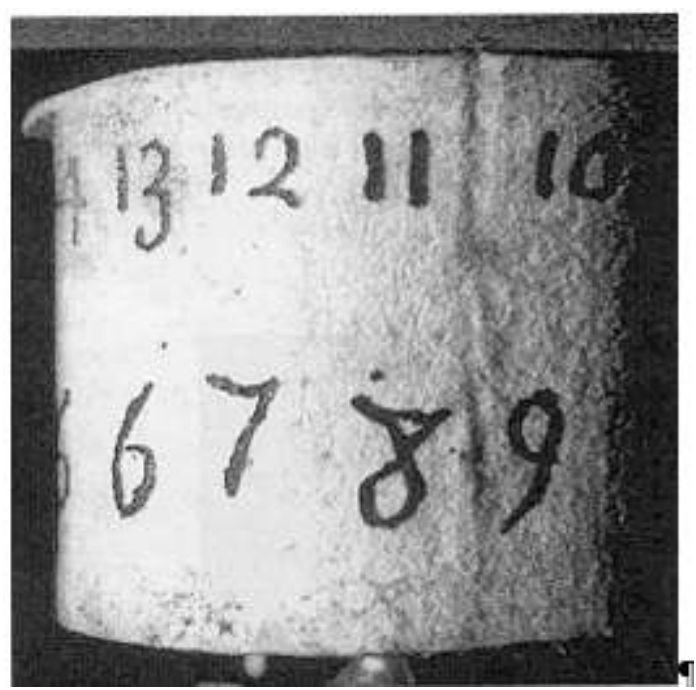

Cl. I.Bonnard. 
Comparaison des graphismes des chiffres sur les rouleaux de la machine « de Marguerite Périer ». Cylindre 8 et 7

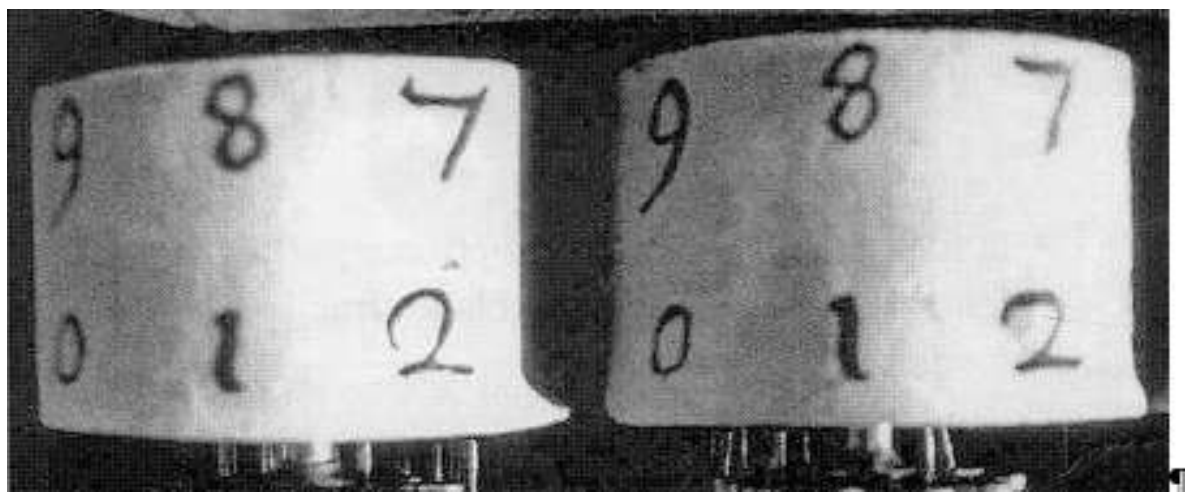

Cl. I. Bonnard

Cylindres 6, 5 et 4 de la machine « de Marguerite Perrier." "

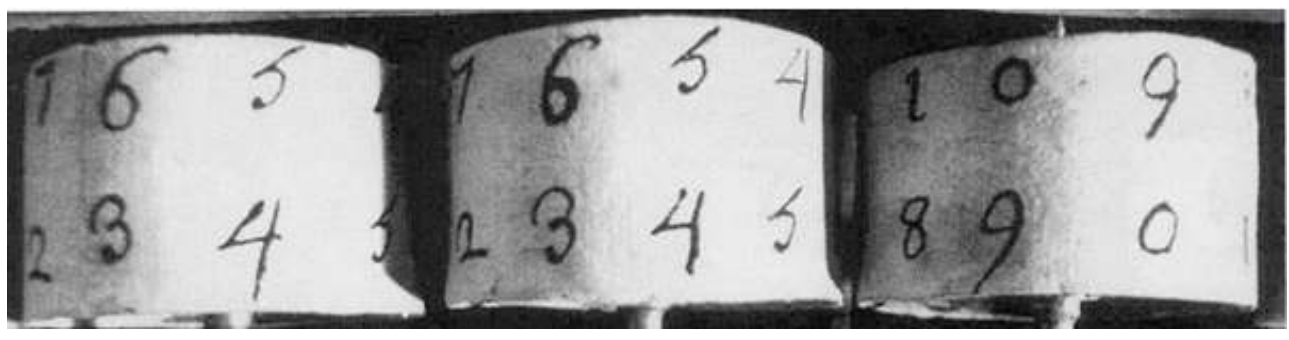

Cl. I. Bonnard

\section{L'avenir}

Les deux Pascalines, restaurées, sont maintenant visibles dans le hall d'entrée du muséum d'histoire naturelle Henri-Lecoq.

Mais l'intérêt de ce genre d'objet est, entre autres, de pouvoir être manipulé pour en comprendre le fonctionnement. Il est évidemment hors de question de sortir ces deux machines pour des manipulations devant le public. Le musée a donc fait réaliser, par un passionné de la région, Pierre Charrier, une copie fonctionnelle qui permet donc les démonstrations.

Prochainement, une nouvelle vitrine sera installée pour mettre en valeur ces deux trésors de notre patrimoine historique et scientifique et la collection de machines arithmétiques du musée.

\section{INDEX}

Mots-clés : pascaline 
AUTEUR

PIERRE PÉNICAUD

Conservateur en chef du patrimoine,

Directeur Du muséum d'histoire naturelle Henri-Lecoq 